Voix et Images

voixetimages

\title{
Auteurs et entreprises dans l'édition littéraire contemporaine au Québec
}

\section{Claude Martin et Vincent Nadeau}

Volume 14, numéro 2 (41), hiver 1989

L’édition littéraire au Québec

URI : https://id.erudit.org/iderudit/200771ar

DOI : https://doi.org/10.7202/200771ar

Aller au sommaire du numéro

Éditeur(s)

Université du Québec à Montréal

ISSN

0318-9201 (imprimé)

1705-933X (numérique)

Découvrir la revue

Citer cet article

Martin, C. \& Nadeau, V. (1989). Auteurs et entreprises dans l'édition littéraire contemporaine au Québec. Voix et Images, 14(2), 225-236.

https://doi.org/10.7202/200771ar d'utilisation que vous pouvez consulter en ligne.

https://apropos.erudit.org/fr/usagers/politique-dutilisation/ 


\title{
Auteurs et entreprises dans l'édition littéraire contemporaine au Québec
}

\author{
Par Claude Martin, Université de Montréal \\ et Vincent Nàdeau, université Laval
}

Notons d'entrée de jeu que les femmes auteures sont si nombreuses au Québec, que nous pourrions mettre au féminin tout ce qui se trouve ici conventionnellement au masculin.

Pour nous, l'édition littéraire est celle qui publie, sous forme de livres ou de brochures, dans des revues ou dans des journaux, de la poésie, des proses poétiques, des romans, des nouvelles ou des contes, des récits, des biographies ou des autobiographies, des correspondances d'auteurs, du thêâtre, des essais, des recueils d'articles, de billets, de maximes, de la critique, des scénarios, de la bande dessinée, ou des textes divers que ne coifferaient pas ces rubriques ou qui en seraient le croisement. À quoi s'ajoutent les éditions critiques. Nous nous demanderons d'abord quelles formes adoptent les rapports des auteurs québécois d'aujourd'hui avec les éditeurs, pour ensuite nous pencher sur les structures de l'industrie de l'édition littéraire au Québec.

Quant aux rapports des auteurs avec les éditeurs, examinons tout de suite deux cas extrêmes, également intéressants, celui des auteurs publiés à l'étranger et celui des auteurs qui se publient eux-mêmes. La simple mention de ces deux catégories indique l'existence d'un phénomène de rejet de la part de certains auteurs, comme de la part de certains éditeurs. Autrement dit, il n'y a pas adéquation entre l'offre des auteurs, et la demande des éditeurs, ce qui est normal en économie de marché et qui pose d'emblée le littéraire dans une logique de produit.

Les auteurs québécois qui écrivent en anglais, ou dans d'autres langues que le français et l'anglais ${ }^{1}$, sont le plus souvent publiés hors du Québec, ou alors à compte d'auteur avec des tirages restreints. L'édition québécoise allophone en effet a eu tendance à disparaître, essentiellement au profit de la métropole ontarienne, et dans une certaine mesure de la région de Vancouver. Mais le

1 En 1987, 86,58 \% des titres enregistrés au dépôt légal du Québec sont en langue française. Bibliothèque nationale du Québec, Statistiques de l'édition au Québec en 1987, Montréal, ministère des Affaires culturelles, 1988, p. 24. 
débouché souhaitable pour un auteur de langue anglaise est naturellement les États-Unis: l'on s'y fait publier si on le peut, court-circuitant au besoin le marché culturel canadian.

D'où il découle que les auteurs québécois de langue française traduits sont publiés à l'étranger, par quoi nous entendons le reste du Canada aussi bien que les États-Unis ou le Japon. Ils sont également publiés à l'étranger, surtout en France, soit lorsqu'il y a cession partielle de droits de la part d'un éditeur québécois, soit lorsqu'il y a co-édition, soit lorsqu'un éditeur de langue française est le seul acquéreur des droits, soit enfin lorsque l'auteur confie son œuvre à des maisons étrangères spécialisées dans le compte d'auteur.

Le nombre des auteurs québécois ${ }^{2}$ publiés en français à l'étranger est assez réduit $^{3}$. Les plus connus sont Gabrielle Roy, Anne Hébert, Jacques Godbout, Claude Jasmin, Marie-Claire Blais, Réjean Ducharme, Antonine Maillet et Yves Beauchemin, sans compter les nombreux poètes qui ont réussi, souvent grâce à des échanges et à des colloques, à s'assurer une certaine diffusion internationale.

Les principaux types de raisons pour lesquelles certains auteurs souhaitent être publiés hors du Québec sont les suivants: refus des éditeurs québécois, rapidité d'acceptation plus grande des éditeurs étrangers, accès au marché francophone, meilleure diffusion au Québec par les représentants d'éditeurs européens, réputation au Québec accrue du fait d'une reconnaissance de l'extérieur. Structurellement, l'industrie française étant extrêmement sélective à l'égard des auteurs québécois, qui d'ailleurs ne sont pas davantage retenus que les wallons, les romands, les maghrébins, les antillais ou les africains noirs, le fait d'être publié en France, même s'il n'entraîne pas automatiquement de succès notables dans le marché francophone, y compris celui de la mère patrie, peut créer, consolider ou mousser une carrière au Québec. C'est un capital symbolique recherché en soi, et qui a très souvent des effets positifs sur les ventes au Québec, à tel point que les éditeurs québécois achètent volontiers lorsqu'ils le peuvent les droits canadiens ou nord-américains des Québécois ainsi attachés à des entreprises externes. On est même allé jusqu'à la piraterie dans le cas de Réjean Ducharme. Ceci explique l'attrait constant qu'exercent faute de mieux les imprimeries ou les «éditeurs» européens qui font la promotion du compte d'auteur.

2 Sauf précision contraire, l'adjectif désignera désormais les auteurs de langue française gravitant dans l'orbite québécoise de l'industrie de l'édition, quelle que soit leur citoyenneté et même s'ils proviennent de régions partiellement francophones du Canada comme l'Acadie, l'Est ontarien ou le Manitoba, la question d'un patrimoine national littéraire dans le cas d'un peuple dépourvu de pays totalement distinct étant particulièrement ardue.

3 En 1983,14,8 \% des auteurs membres de l'Union des écrivains québécois publiaient généralement des œuvres à l'étranger. Annexe. Enquête auprès des auteurs québécois. Tableaux statistiques, Québec, ministère des Affaires culturelles, 1986, p. IV-21. 
Au Québec, l'auto-édition représente une part importante des titres publiés par les écrivains ${ }^{4}$. Une association des auteurs-éditeurs a même existé pendant un certain temps, dans le but d'assurer un soutien technique à la fabrication et à la distribution et de constituer un groupe de pression reconnu. Les raisons de cette prolifération sont à explorer de divers côtés: recherche d'identité personnelle ou collective, généralisation de l'éducation secondaire, valorisation des arts, marginalisation des plus jeunes, chômage endémique, stabilité relative des coûts de production et plus grande capacité de fabrication, disponibilité accrue de machines à écrire, de photocopieuses, d'ordinateurs et d'imprimantes, augmentation sensible des ateliers d'écriture à l'école et hors de l'école, augmentation générale de la production et de la consommation d'imprimés, augmentation du nombre d'éditeurs et paradoxalement monopolisation par une classe d'âge des leviers de sélection, quasi absence d'éditeurs en région, professionnalisation du métier d'écrivain et donc imposition de barrières supplémentaires à l'entrée, politiques de subventions favorisant la concentration et l'expérience, etc.

Venons-en maintenant à ceux des auteurs québécois qui sont publiés par des éditeurs québécois. La forme de leurs rapports varie selon plusieurs paramètres. Quand il s'agit des journaux ou de certaines revues, l'auteur pourra être salarié, et alors il devra négocier avec les détenteurs de ses droits une éventuelle publication en livre. Si l'auteur est pigiste, dans le meilleur des cas il touchera une somme forfaitaire, généralement peu élevée. Il lui arrivera aussi de ne rien toucher, comme dans le cas des revues «qui commencent» et des revues régionales, culturelles ou autrement marginales, la rémunération du travail étant ici purement symbolique. Toutefois, le pigiste reste propriétaire de ses droits, la valeur monétaire en étant négligeable.

S'agissant des éditeurs de livre, les rapports peuvent être, dans un petit nombre de cas, médiatisés par des agents littéraires (qui peuvent être des avocats), par une association comme l'Union des écrivains québécois ou par les décisions réglementaires ou législatives adoptées par les gouvernements de Québec ou d'Ottawa en matière de statut de l'écrivain, de droit d'auteur ou d'aide financière. Ces dernières incidences jouent bien entendu aussi, dans une moindre mesure, pour les rapports avec les éditeurs de journaux ou de revues. Toute intervention de caractère collectif ou gouvernemental peut entrainer une réaction corporatiste de nature analogue de la part des éditeurs regroupés par exemple dans l'Association des éditeurs canadiens.

Dans la pratique la plus courante, les auteurs se retrouvent seuls face à leur éditeur, sauf en cas d'édition coopérative, de regroupement d'auteurs pour fonder une nouvelle maison d'édition (ex.: les Quinze) ou une revue, de participation à des comités de lecture ou à des jurys ou de direction littéraire (ex.: Gilbert La Rocque chez Québec/Amérique ou Victor-Lévy Beaulieu dans

4 En 1983, 36,9\% des auteurs membres de l'Union des écrivains québécois avaient déjà publié à compte d'auteur. Enquête auprès des auteurs québécois, op. cit., p. IV-17. 
ses entreprises successives ou Gaston Miron à l'Hexagone). Un cumul systématique des fonctions d'actionnaire, de directeur général, de directeur littéraire et d'auteur peut à la limite constituer une forme déguisée et subventionnée d'auto-édition.

Lorsqu'ils agissent isolément, les auteurs tentent de choisir leur éditeur, ou très exceptionnellement sont choisis par eux, en fonction de leur notoriété politique, artistique ou même littéraire ou en fonction de leur compétence à traiter un sujet déterminé par l'éditeur. Un auteur à ses débuts peut envisager diverses stratégies pour frapper à la porte des éditeurs. Soit, en guise d'exploration de la demande, il utilisera classiquement la poste pour envoyer un maximum de copies de son œuvre à un maximum d'éditeurs, soit, en fonction de sa connaissance des catalogues et des auteurs, de la réputation, etc., il choisira quelques-uns seulement, soit, à cause d'une parfaite connaissance du marché ou d'une décision de caractère personnel ou artistique, il n'enverra qu'à un seul, recommençant ou non le processus en cas de refus. L'idéal pour un auteur est naturellement d'avoir des propositions de plus d'un éditeur, ce qui permet d'envisager une meilleure négociation de contrat et qui peut donner plus de poids à son avis concernant la présentation du livre, le prix de vente, la publicité, les relations avec les mass médias, la distribution, etc. Il n'est pas impensable non plus qu'un auteur patient écrive une simple lettre à un ou plusieurs éditeurs pour faire part de projets réalisés ou non, soumettant une idée, un résumé, un sommaire, un chapitre...

L'auteur débutant peut rejeter cette approche longue et indirecte pour tenter une vente plus personnelle et plus immédiate de ses droits. S'il n'a pas déjà de relations particulières avec le milieu de l'édition, il pourra songer à demander un rendez-vous au président-directeur général, au directeur littéraire, aux conseillers littéraires, à quelque membre des comités de lecture ou s'adresser pour conseils ou recommandations à un employé quelconque, ou à des amis, de la maison. Il pourra encore rechercher le parrainage d'un critique influent ou d'une célébrité. Ou il pourra tenter de percer dans un autre domaine, connexe ou non, pour se faire agréer d'un éditeur.

Quelle que soit sa démarche, l'auteur débutant ne peut guère espérer autre chose qu'un pourcentage sur les ventes futures de son ouvre, ce qui comme chacun le sait se réduit presque toujours à peu de choses, à moins d'un succès inespéré. S'il accepte un montant forfaitaire, la rémunération est plus immédiatement palpable mais d'autant plus mince, relativement, que le nombre d'exemplaires vendus est plus grand. Les avances sur droits sont peu fréquentes au Québec et sont habituellement réservées aux écrivains non seulement chevronnés mais qui se vendent bien ou aux personnages très célèbres. On comprend que plus un auteur a eu de succès, plus il pourra exiger un contrat avantageux, par exemple obtenir des engagements fermes en matière de publicité, une augmentation de son pourcentage en proportion de l'augmentation des ventes, etc. Un auteur à succès peut également entretenir des rapports privilégiés avec les relations publiques et les services commerciaux de sa maison. $S$ 'il y a désaccord, il peut menacer de changer d'éditeur ou en changer 
réellement. Néanmoins, débutant ou pas, l'auteur doit savoir qu'il peut exister des différences significatives entre les contrats, selon l'habileté et la détermination de chacun dans la négociation.

Après la signature du contrat, la suite des rapports avec l'éditeur reste à déterminer de gré à gré, en fonction certes des personnalités respectives, mais aussi en fonction de l'enthousiasme que l'auteur apporte aux aspects connexes de son métier: juridique, administratif, comptable, commercial, publicitaire. Il pourra demander des comptes d'autant plus exacts à son éditeur qu'il l'aura appuyé moralement et concrètement à toutes les étapes, depuis la correction des épreuves jusqu'aux entrevues à la radio, à la télévision, et dans les journaux. Un auteur qui paie de sa personne ne peut qu'être bien vu de ses partenaires. Et comme un éditeur ne peut pas envisager de faire de promotion spéciale à plus de quelques titres par année... Ainsi, non seulement il y a concurrence entre les auteurs avant que leur œuvre soit retenue, mais aussi après, au sein d'une même maison. Il est par ailleurs certain que pour un auteur de métier, étant donnée la concentration démographique, le regroupement des maisons d'édition et des librairies à Montréal, de même que celui des mass médias, il est plus efficace, et moins coûteux en déplacements et en téléphones, d'habiter la région métropolitaine. La plupart l'ont compris. D'autant que la variété et le nombre des emplois à temps partiel ou à temps complet sont beaucoup plus grands à Montréal, la majorité des auteurs, littéraires au sens défini plus haut, ne vivant pas de leur plume.

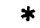

* *

Les relations entre les auteurs et les éditeurs constituent le premier maillon d'une chaîne qui permet la communication entre les auteurs et les lecteurs. Cette chaîne est désormais structurée selon le modèle des industries culturelles: les créateurs confient leurs œuvres à des industriels qui l'éditent, qui exercent un choix parmi les œuvres proposées et qui reproduisent l'œuvre sur un support qui la rend disponible pour un vaste auditoire. Dans le cas de la littérature, ce support est l'imprimés. Une partie de la production prend le chemin de la presse périodique où elle s'intègre dans un contenu essentiellement informatif. La plus grande partie prend la forme de livres ou de périodiques spécialisés à faible tirage. La littérature fonctionne alors selon le modèle des industries culturelles non publicitaires qui vendent leur produits aux consommateurs ${ }^{6}$.

L'édition de livres littéraires est imbriquée dans l'industrie (générale) de l'édition de livre et il n'est pas possible de distinguer parfaitement ces deux domaines. L'industrie de l'édition est elle-même imbriquée dans l'ensemble des

5 Nous pourrions ouvrir ici un débat sur le cinéma et la télévision comme littérature...!

6 Par opposition aux industries culturelles publicitaires qui vendent l'attention des auditoires aux annonceurs. 
industries du loisir et de l'information, industries organisées au niveau mondial et dominées par des conglomérats transnationaux géants. Pourtant, il y a un monde entre une petite maison d'édition de poésie et un conglomérat multimédia. Mais cette distinction n'est pas particulière au monde littéraire. Dans toutes les industries, on trouve des petites firmes innovatrices qui prennent de grand risques avec des produits nouveaux et des grandes entreprises qui dominent le marché avec une production correspondant aux «goûts moyens». Ce qui est différent ici, c'est le produit, la littérature, une marchandise volontairement chargée de sens, un produit de l'esprit dont l'utilité est justement de ne pas être «fonctionnel» ${ }^{7}$. Dans ces conditions, il n'est pas étonnant qu'une partie de l'industrie refuse de se voir comme une industrie et préfère se considérer comme une œuvre culturelle.

Ce qui ne nous empêche pas de regarder l'édition littéraire d'un point de vue économique et de la considérer comme une industrie puisqu'on y dépense des ressources et qu'on y produit des marchandises que des consommateurs achètent. Regardons le premier tableau (p. 231) intitulé «Les structures ${ }^{8}$ de l'édition de livres littéraires». Il présente, en la simplifiant, l'organisation actuelle de l'industrie au Québec. Nous avons dû simplifier le schéma en éliminant certains acteurs ou certaines relations afin de ne pas le surcharger et d'en conserver la lisibilité.

L'édition de livres littéraires est aujourd'hui réalisée par une série d'entreprises spécialisées dans une ou quelques fonctions. Il n'en a pas toujours été ainsi. Au XVe siècle, l'imprimeur se charge de toutes les opérations nécessaires pour rendre le livre jusqu'au lecteur; il est imprimeur, éditeur et libraire. Au XVIIIe siècle, l'éditeur se distingue de l'imprimeur et du libraire et il devient le premier responsable de la parution du livre. Au $\mathrm{XX}^{\mathrm{e}}$ siècle, la fonction de distribution prend une importance de plus en plus grande. Cette spécialisation des fonctions n'empêche cependant pas certaines entreprises de les réunir sous une direction unifiée (c'est-à-dire de pratiquer l'intégration verticale).

Deux types de marchandise circulent entre les entreprises: les droits de reproduction et les livres ${ }^{9}$. Ainsi, notre schéma montre que les éditeurs peuvent acheter les deux types de produits: de fait, environ le quart des recettes des éditcurs (littéraires ou non) du Québec proviennent de leurs activités d'agent

7 Est littéraire toute cuvre qui n'est pas un outil, mais une fin en soi. Est littéraire toute lecture non fonctionnelle, c'est-à-dire satisfaisant un besoin culturel non utilitaire. R. Escarpit, Sociologie de la littérature, 6c éd., Paris, PUF, 1978, p. 21.

8 Pour une industrie donnée, la structure reflète les forces stables qui caractérisent l'environnement des firmes considérées [...]. R.E. Caves cité dans J.M. Chevalier, l'Économie industrielle en question, Paris, Calmann Lévy, 1977, p. 18. Selon les auteurs, le terme structure se rapporte à divers phénomènes (nombre de firmes, intégration verticale ou conglomérale, barrières à l'entrée, courbe des coûts, etc.). Nous ne retenons ici qu'un aspect de ces structures, la division du travail.

9 On pourrait ajouter à ces produits certains produits intermédiaires comme des fichiers ordinolingues ou des matrices d'imprimerie. 
exclusif, activités où ils agissent comme diffuseur pour les livres d'un autre éditeur ${ }^{10}$. Il s'agit alors généralement d'importations. Pour leur part, les auteurs vendent généralement les droits de reproduction de leurs œuvres; mais ceux qui pratiquent l'auto-édition vendent aussi des livres (un phénomène que nous n'avons pas indiqué sur notre schéma).

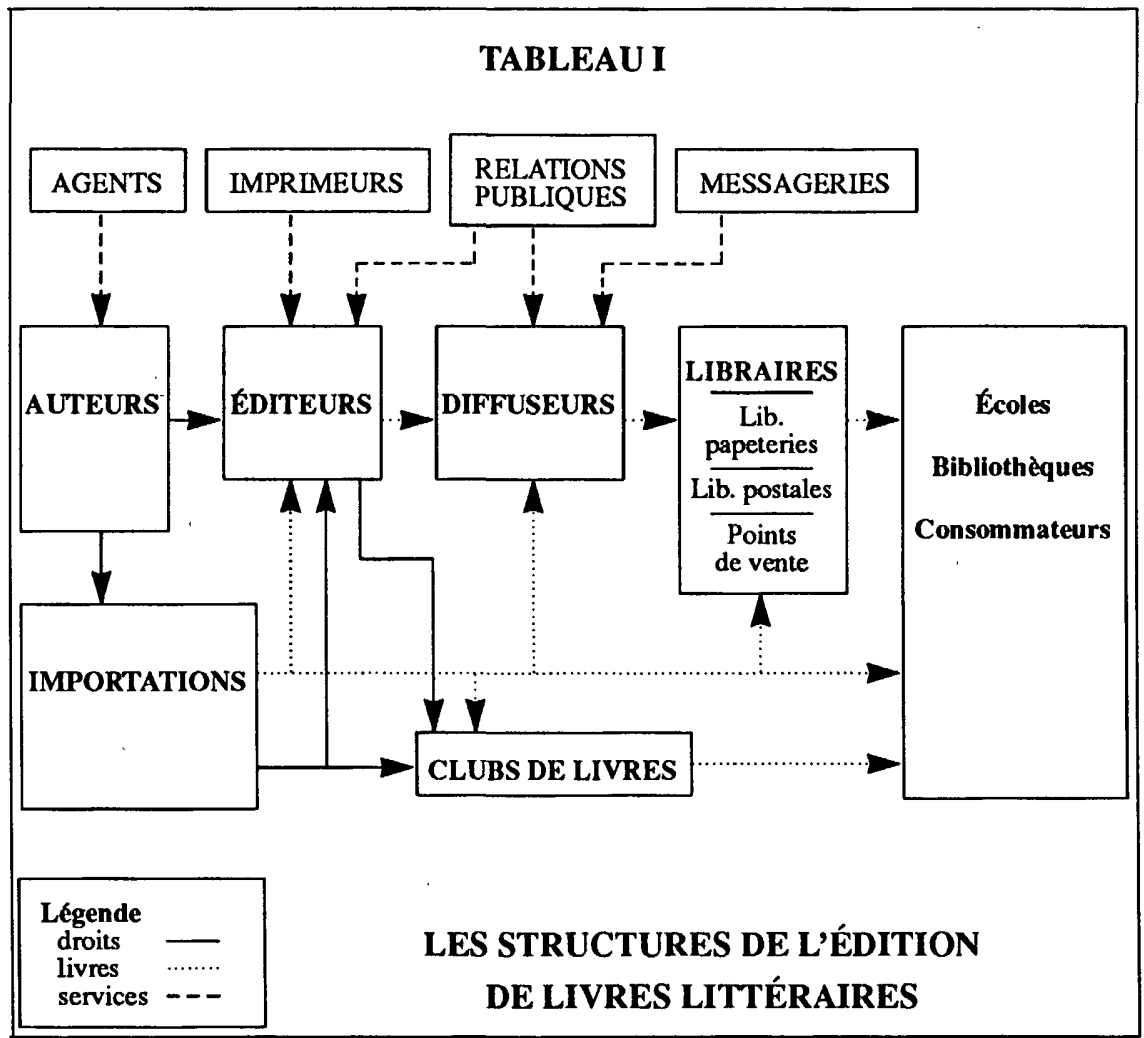

Regardons celui-ci par la fin. L'industrie dessert trois types de client: les consommateurs individuels qui paient pour se procurer un livre, les bibliothèques publiques, scolaires, collégiales et universitaires et le système scolaire qui demande la lecture de certaines œuvres littéraires dans ses programmes ${ }^{11}$. Le marché institutionnel représente environ $30 \%$ du total ${ }^{12}$. Les écoles primaires

10 Nous ne pouvons affirmer si cette proportion est aussi élevée dans le cas des livres littéraires.

11 Notons que nous ne parlons pas ici des manuels scolaires, un tout autre marché.

12 Drouin, Paquin \& Associés Ltée, Étude visant à déterminer une stratégie de développement des marchés de l'édition en langue française au Canada et en 
et secondaires prêtent en général les livres aux écoliers, mais les collèges et les universités demandent aux étudiants de se les procurer en librairie. On peut estimer que le marché du livre au Québec représente environ 350 millions \$ en 1988 (au niveau des prix de détail) ${ }^{13}$. De cette somme, environ $40 \%$ va à ce que les statistiques appellent la «littérature générale (tradebooks)». Cette dernière catégorie comprend les livres fonctionnels (livres pratiques, scientifiques, etc.) et la littérature. Certaines enquêtes permettent de penser que ces deux catégories connaissent une popularité équivalente ce qui laisserait (sous toutes réserves) un marché de 70 millions \$ (environ 5 millions d'exemplaires) pour les livres «littéraires». Il faut cependant manipuler les données précédentes avec prudence: le terme «littéraire» est ici utilisé dans son sens le plus large et les données portent sur toutes les ventes de livres (incluant les livres importés et les ventes qui ne passent pas par les librairies).

Environ la moitié des achats de livre se font en librairie. La grande majorité des librairies sont des librairies-papeteries ${ }^{14}$. Les institutions publiques doivent s'approvisionner auprès des librairies agréées. Ces dernières obtiennent ce privilège contre certaines obligations dont celle de conserver un fonds d'ouvrages québécois. En 1986, on comptait 175 de ces librairies parmi les quelques 250 librairies au Québec. Les librairies agréées sont généralement indépendantes mais les chaînes ${ }^{15}$ réalisent environ la moitié des ventes. Les points de vente (pharmacies, dépanneurs, tabagies, grands magasins, etc.) réalisent environ le quart des ventes avec essentiellement des best-sellers et des romans sentimentaux.

Entre l'éditeur et le libraire, sont apparus les diffuseurs (aussi appelés distributeurs) qui font office de grossistes pour les détaillants que sont les libraires. Certains sont étroitement liés à un ou des éditeurs; d'autres travaillent pour le compte de plusieurs. On compte une vingtaine de diffuseurs au Québec ${ }^{16}$. Les fonctions des diffuseurs varient selon les cas. Au Québec, tous disposent d'un entrepôt afin de réaliser les opérations de regroupement et d'aiguillage des livres vers les détaillants. Mais certains (parmi les plus importants) cessent là leurs opérations alors que d'autres se chargent aussi de la promotion des livres auprès des libraires et du public. Peu de diffuseurs distribuent physiquement leurs livres; ils les confient plutôt à des messageries générales (des services de courrier comme on les nomme souvent). Lorsqu'un livre sort du circuit des

France - Situation sur les marchés canadiens, rapport remis à la Société de développement du livre et du périodique.

13 Plusieurs des données qui suivent proviennent (parfois après indexation) de C. Martin, le Poids économique des industries culturelles non publicitaires, rapport remis au ministère des Affaires culturelles, 1986.

14 G. Hardy, J.-P. Sylvestre et S. Jolin, Livres et librairies agréés. Chiffres à l'appui, vol. III, no 6, 1986.

15 Les librairies qui ne sont pas indépendantes et qui font partie d'un groupe financier.

16 Pour une liste des principaux diffuseurs de best-sellers, voir C. Martin, «Comme des petits pains chauds. Essai d'économie industrielle du best-seller en français au Québec», Communication information, vol. VII, n 3, 1985, p. 106-127. 
librairies et se diffuse dans les points de vente, il passe alors par les messageries de presse, seules capables de desservir rapidement un réseau si important ${ }^{17}$.

Selon les données les plus récentes de Statistiques Canada, en 1984, le Québec comptait 71 éditeurs privés ${ }^{18}$. Pour sa part, l'Union des écrivains québécois présente une liste de 88 éditeurs présents au Québec en 198819. Parmi ces derniers, nous en trouvons 29 qui affirment publier de la littérature. Dans le tableau II, «Les principaux éditeurs québécois publiant de la littérature» (p. 234), nous présentons une liste des plus importants parmi ces éditeurs. Nous ne disposons pas de données sur le nombre d'ouvrages littéraires publiés par ces éditeurs; nous avons donc classé les éditeurs selon le nombre total de titres (littéraires ou non) publiés. Cette donnée présente un intérêt car elle indique le poids économique de l'éditeur. Un éditeur peut n'avoir publié que quelques titres littéraires mais ces titres profitent d'un pouvoir économique plus grand que ceux d'un petit éditeur spécialisé dans la littérature. On notera dans ce tableau la présence de deux importants éditeurs de journaux, la Presse et Quebecor. En plus de passer par le circuit des librairies, le premier agit comme une librairie postale auprès de ses abonnés alors que le second dispose d'une des deux messageries de presse du Québec et d'une entreprise de diffusion de livres. Deux maisons font partie du groupe Sogides, le plus important éditeur privé au Québec. On trouve aussi sur cette liste deux presses universitaires qui n'ont pas comme fonction principale de publier de la littérature. Parmi les plus importants éditeurs, on voit Guérin, surtout connu pour ses manuels scolaires et récemment arrivé dans le marché du livre littéraire pour le grand public. Enfin, soulignons que la maison Leméac vient de connaître de sérieuses difficultés financières. En 1987, les éditeurs du Québec ont produit 4219 livres et 2238 brochures ${ }^{20}$. Dans ces nombres, on compte 486 livres et 221 brochures littéraires ${ }^{21}$ (dont 193 brochures dans la catégorie «littérature pour la jeunesse»). Parmi les livres, il y a 183 romans et 134 livres de poésie. Bien que nous n'ayons pas de données précises sur le sujet, il semble que les éditeurs du Québec font généralement imprimer leurs livres au Québec. L'industrie québécoise de l'imprimerie est très dynamique et les spécialistes de l'impression de livre y occupent une place stable22.

Retournons au schéma sur les structures de l'édition. Les diffuseurs et les éditeurs font parfois appel aux services des firmes de relations publiques. Il s'agit ici surtout de spécialistes des relations avec les médias qui doivent

17 Il ne reste que deux messageries de presse au Québec, Benjamin (indépendant) et Dynamiques (du groupe Quebecor).

18 Éditeurs ayant publié ou réimprimé un livre et/ou ayant vendu pour plus de $50000 \$$ de livres pendant l'année. Statistiques Canada, \# 87-525.

19 Excluant le Gouvernement du Québec et incluant l'Université d'Ottawa. UNEQ, le Métier d'écrivain, 2e éd., Montréal, Boréal, 1988, chap. 5.

20 Bibliothèque nationale du Québec, op. cit., Une brochure comporte moins de 49 pages. Notons que les réimpressions ne sont pas comptées dans ce système.

21 Poésie, théâtre, roman, conte et nouvelle, correspondance, essai, littérature pour la jeunesse.

22 Signalons l'imprimerie Gagné et celle de Payette \& Simms parmi les plus connues. 


\section{TABLEAU II}

\section{Les principaux éditeurs québécois* publiant de la littérature** selon le nombre de titres (littéraires ou non) publiés (jusqu'en 1988)}

\begin{tabular}{|c|c|c|}
\hline Rang & Éditeur & Nombre de titres*** \\
\hline 1 & Fides & 1967 \\
\hline 2 & Guérin & 1600 \\
\hline 3 & 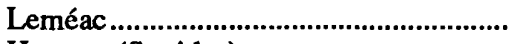 & 900 \\
\hline 4 & 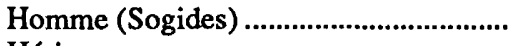 & 814 \\
\hline 5 & 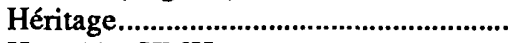 & 800 \\
\hline 6 & 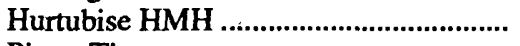 & 645 \\
\hline 7 & 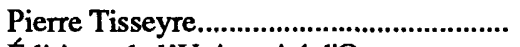 & 560 \\
\hline 8 & Editions de l'Université d'Ottawa ........... & 550 \\
\hline 9 & Quebecor & 535 \\
\hline 10 & 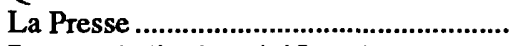 & 505 \\
\hline 11 & Presses de l'université Laval.................... & 497 \\
\hline 12 & 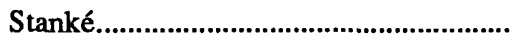 & 423 \\
\hline 13 & 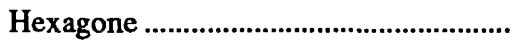 & 400 \\
\hline 14 & 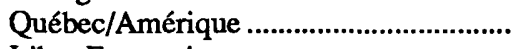 & 372 \\
\hline 15 & 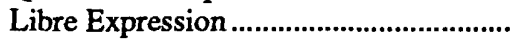 & 350 \\
\hline 16 & 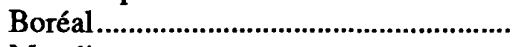 & 250 \\
\hline 16 & 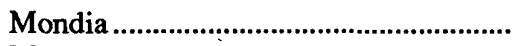 & 250 \\
\hline 16 & 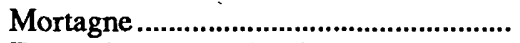 & 250 \\
\hline 17 & Entreprises culturelles inc............................ & 245 \\
\hline 18 & VLB & 235 \\
\hline 19 & 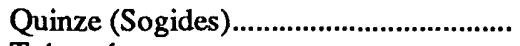 & 227 \\
\hline 20 & 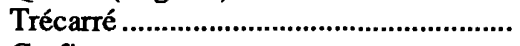 & 200 \\
\hline 21 & Graficor & 189 \\
\hline 22 & 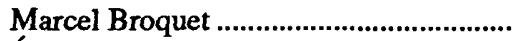 & 151 \\
\hline 23 & 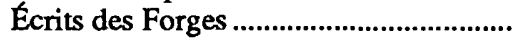 & 120 \\
\hline 23 & 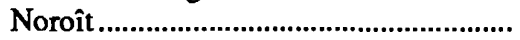 & 120 \\
\hline 24 & 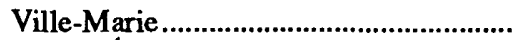 & 116 \\
\hline 25 & 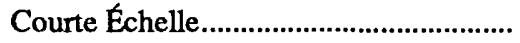 & 100 \\
\hline 25 & Prise de parole & 100 \\
\hline
\end{tabular}

Notes: * Selon la source, cette liste n'est pas exhaustive; nous avons retenu les éditeurs de 100 titres ou plus. L'Université d'Ottawa et Prise de parole sont situées en Ontario.

** Nous avons retenu les éditeurs qui, selon la source, indiquent une ou des «disciplines» littéraires (littérature, roman, poésie, théâtre, essai, littérature pour la jeunesse, best-sellers, etc.).

*** La proportion de titres littéraires peut être faible pour certains éditeurs.

Source: Compilé à partir de la liste des éditeurs dans UNEQ, le Métier d'écrivain, 2c éd., Montréal, Boréal, 1988, chap. 5. 
s'assurer que les bons journalistes reçoivent les bons livres et que les auteurs obtiennent leur part de l'attention des médias. Les éditeurs et les diffuseurs se chargent souvent de ce travail, mais l'appel aux firmes spécialisées se développe même si cette activité ne représente pas, pour le moment, une part importante des dépenses des éditeurs et des distributeurs ${ }^{23}$.

Parallèlement au circuit éditeurs-diffuseurs-libraires, on trouve les clubs de livres. Ceux-ci vendent directement au consommateur des livres qu'ils éditent (contrairement aux librairies postales qui vendent des livres édités par d'autres). Il s'agit généralement de livres rédités après avoir connu le succès en librairie. Les deux plus importants clubs de livres qui vendent des livres littéraires en français au Québec sont Québec Loisirs, lié au groupe allemand Bertelsmann et Laffont, un éditeur français célèbre pour ses best-sellers. Leurs catalogues comportent une forte proportion de titres importés (qui peuvent être imprimés au Québec, une possibilité que notre schéma n'indique pas).

Ayant ainsi remonté le fleuve de la littérature, nous nous retrouvons aux sources, chez les auteurs. Lors du recensement de 1981, 7300 résidents du Québec ont indiqué, comme métier, écrivain ou rédacteur. Cette catégorie comprend un bon nombre de journalistes et de rédacteurs techniques ou publicitaires. Par ailleurs, plus de 7000 personnes réclament des droits d'auteurs selon le système de compensation pour les photocopies administré par l'Union des écrivains québécois (UNEQ). Les différentes associations d'auteurs du Québec ${ }^{24}$ comptent environ un millier de membres. Pour sa part, l'UNEQ compte environ 425 membres, un nombre généralement accepté comme proche du nombre d'auteurs de livre actifs au Québec.

Parmi les membres de l'UNEQ qui ont répondu en 1983 à un sondage du ministère des Affaires culturelles $25,39 \%$ sont des femmes. Près de la moitié disent écrire régulièrement de la poésie et des romans. Seulement $28 \%$ de ces auteurs pratiquent le métier d'auteur à plein temps. Les trois quarts ont un second métier connexe à celui d'écrivain et pour la moitié de ces derniers, il s'agit de l'enseignement. En 1983, $34 \%$ de ces auteurs gagnaient plus de $40000 \$$ par année alors que $44 \%$ gagnaient moins de $10000 \$$. Mais une partie seulement de ces revenus provient de l'activité d'auteur: $70 \%$ des auteurs reçoivent moins de $25 \%$ de leurs revenus du métier d'auteur.

Il nous reste une case dans notre schéma: les importations. Nous avons déjà vu que certains auteurs québécois sont édités à l'étranger. Mais il est bien évident que la très grande majorité des livres et des périodiques à contenu littéraire importés au Québec contiennent des textes d'auteurs non québécois. Le Québec importe des livres des États-Unis, de l'Angleterre, mais surtout, de

23 Notons que le milieu fait très peu appel aux agences de publicité.

24 L'UNEQ, la Société des auteurs, recherchistes, documentalistes et compositeurs (SARDEC), l'Association des traducteurs littéraires, la Société professionnelle des auteurs compositeurs québécois (SPAQ), le Centre d'essai des auteurs dramatiques.

25 Ces données et celles qui suivent proviennent de l'Enquête auprès des auteurs québécois, op. cit., partie IV. 
la France. Les éditeurs français nous y présentent des auteurs français ou francophones (incluant des auteurs québécois) et des auteurs de diverses langues traduites en français dont une forte proportion d'auteurs américains. La France sert alors de relais pour la diffusion de la culture américaine au Québec! Les éditeurs québécois tentent aussi d'obtenir les droits mondiaux de certains textes mais il semble qu'il soit plus facile d'importer des livres plutôt que des droits!

Selon les hypothèses, les définitions et les sources, on peut estimer que les livres littéraires québécois (édités au Québec et/ou écrits par des auteurs québécois) représentent entre 5 et $25 \%$ du marché. Si on regarde ce phénomène sur une longue période, disons depuis 1945, on peut estimer que les auteurs et l'industrie littéraire du Québec occupent une place de plus en plus grande sur le marché. Il ne s'agit pas de dire ici que la situation est satisfaisante, que les auteurs gagnent bien leur vie ou que l'État devrait cesser de soutenir ce secteur. Mais il faut constater l'émergence d'auteurs et de professionnels de l'édition désormais capables de défendre et d'agrandir leur territoire dans un secteur caractérisé par une division du travail typique des industries culturelles non publicitaires et par une mondialisation des structures de production. À ce chapitre, la performance de la littérature nous semble meilleure que celle des industries de l'enregistrement et du cinéma. Il suffit de comparer les listes de best-sellers avec celles du box-office et celles des palmarès pour s'en convaincre. 